\title{
Heartbeat: Biomarkers and pulmonary artery hypertension
}

\section{Catherine M Otto}

Pharmacologic treatment for pulmonary arterial hypertension (PAH) remains suboptimal and mortality rates are still high, even with pulmonary vasodilator therapy. In addition, we have only an incomplete understanding of the pathobiology of $\mathrm{PAH}$, which is characterized at the tissue level by fibrosis, hypertrophy and plexiform remodeling of the distal pulmonary arterioles. Novel therapeutic approaches that might target pulmonary vascular remodeling, rather than pulmonary vaso-reactivity, require precise patient phenotyping both in terms of clinical status and disease subtype. However, current risk stratification models are cumbersome and not precise enough for choosing or assessing the results of therapeutic intervention. Biomarkers used in patients with left heart failure, such as troponin-T and N-terminal pro-B-type natriuretic peptide (NT-proBNP) are elevated in PAH patients but tend to simply reflect increased circulating plasma volumes and elevated right heart pressure, rather than conveying information about disease mechanism.

In this issue of Heart, Calvier and colleagues (see page 390) propose galectin-3 as a useful biomarker in PAH. The rationale for this hypothesis is that elevated aldosterone levels induce an increase in serum levels of galectin-3, a $\beta$-galactoside-binding lectin expressed by circulating myocytes, endothelial cells and other cardiovascular cell types. Among other effects, activation of the aldosterone/galactin-3 pathway promotes fibrosis, suggesting that elevated levels will correlate with the severity of $\mathrm{PAH}$ due to increased pulmonary arteriolar remodeling. To test this hypothesis, serum levels were measured in a total of 57 patients - 41 with idiopathic PAH (iPAH) and 16 with PAH associated with a connective tissue disorder (CTD). The magnitude of elevation in serum levels of aldosterone, galectin-3 and NT-proBNP each correlated with the severity of PAH. However, as shown in figure 1, although serum levels of galectin-3 were elevated in both $\mathrm{PAH}$ and PAH-CTD patients, aldosterone was elevated only in those with iPAH. In addition, elevated vascular cell adhesion molecule 1 (VCAM-1)

Correspondence to Professor Catherine M Otto, Division of Cardiology, University of Washington, Seattle, WA 98195, USA; cmotto@u.washington.edu and proinflammatory, anti-angiogenic interleukin 12 (IL-12) in were elevated only in PAH-CTD patients, not in those in $\mathrm{PAH}$. These data suggest that aldosterone and galectin-3 can be used as biomarkers "in tandem" that reflect both the severity and cause of PAH.

In the accompanying editorial, Maron (see page 335) summarizes the knowledge gaps in PAH and concludes: "Taken together, Calvier and colleagues provide a key contribution to an underdeveloped area of pulmonary vascular medicine and in doing so identify galectin-3/aldosterone as promising biomarker(s) for informing both disease pathobiology and clinical status in PAH. The rationale of this pursuit in PAH was based, in part, on lessons earned from left heart failure in which the importance of systemically circulating vasoactive factors to clinical trajectory is well established. In this regard, the current work not only develops a
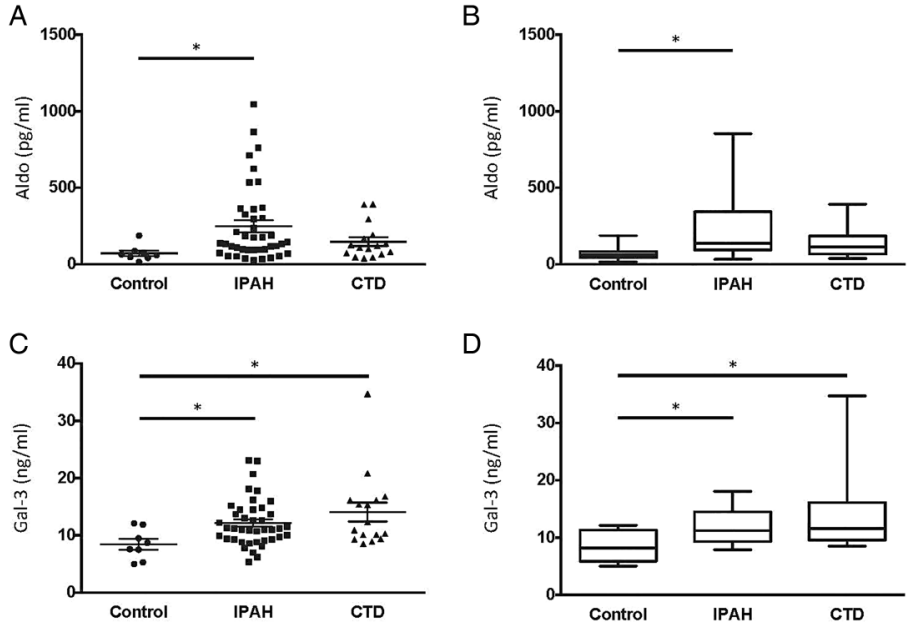

D
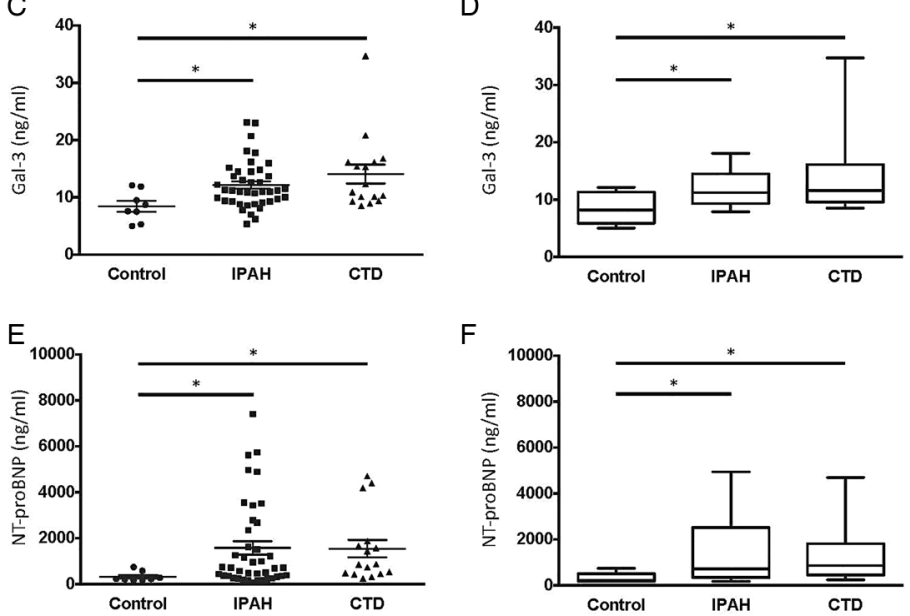

Figure 1 Galectin-3, Aldosterone, and NT-proBNP plasma concentrations are elevated in PAH patients vs. controls. The scatter plots on the left and the box and whiskers plots on the right provide data for aldosterone (A and B), galectin-3 (C and D), and NT-proBNP (E and F) plasma concentrations in control subjects $(n=8)$, patients with idiopathic pulmonary hypertension (IPAH, $n=41$ ), and PAH associated with connective tissue disease (CTD; $n=16)$. The scatter plots show the mean $\pm S E M$; the box and whiskers plots show the median with interquartile range $\pm 10-90$ percentile. ${ }^{*} \mathrm{p}<0.05,{ }^{* *} \mathrm{p}<0.01$. 


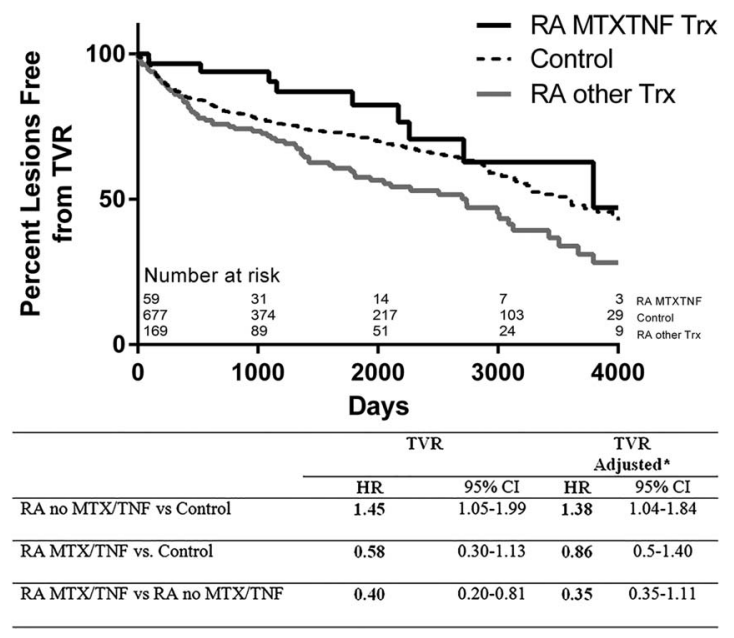

Figure 2 Target Vessel revascularization amongst lesions in patients with Rheumatoid Arthritis not treated with methotrexate or Tumor Necrosis Factor Alpha inhibitors compared to those with Rheumatoid Arthritis treated with methotrexate or Tumor Necrosis Factor Alpha inhibitors versus matched controls. MTX/TNF=treated with methotrexate or tumor necrosis factor alpha inhibitor at discharge from index PCl, MTX/TNF $n=59$; no MTX/TNF $n=169$; Control $n=677^{*}$ adjusted for stent length, overlapping stents, bifurcation stenting, bare metal stent use and intervention on saphenous vein graft in cox proportional hazards model.

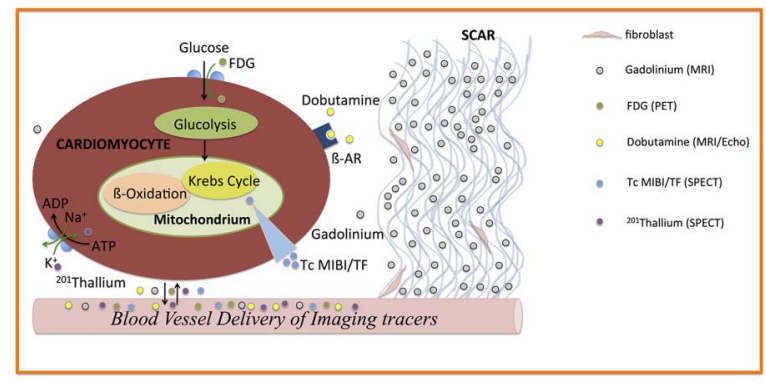

Figure 3 Pathophysiological targets of differing imaging modalities, demonstrating targets for tracers/contrast agents/pharmacotherapy used in SPECT, PET, MRI and echocardiography to assess myocardial viability. Adapted from Schuster et al., J Am Coll Cardiol 2012;59:359-70.

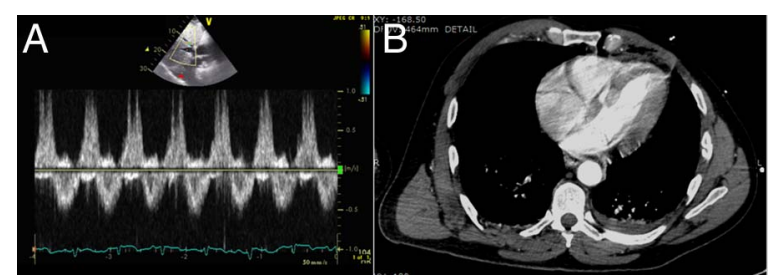

Figure 4 (A) Hepatic vein pulse wave Doppler study from subcostal window. (B) CT of chest with contrast.

patients undergoing PCI. Overall, repeat TVR occurred in 39\% of RA patients compared to $31 \%$ of controls (adjusted HR $1.1595 \%$ CI $0.82-1.6$ ), but the hazard ratio was significant when only events occurring more than one year after the initial PCI were considered (adjusted 1.55 95\% CI 1.12-2.14). Treatment with disease modifying agents for RA modulated this effect with similar rates of TVR in treated RA patients compared to controls (figure 2). In contrast, RA patients not receiving disease modifying therapy were at increased risk of repeat TVR (adjusted HR 1.38 95\% CI 1.041.84). The authors conclude that systematic inflammation in RA patients is associated with an approximately 50\% increased risk of recurrent coronary disease after PCI. However, this increase in risk is not seen in RA patients receiving treatment with disease modifying agents, including methotrexate and/or tumor necrosis factor (TNF $\alpha$ ) inhibitors, supporting the need for aggressive antiinflammatory therapy for secondary prevention of recurrent coronary disease, in RA patients. The authors further speculate that "Patients with systemic lupus erythematosus, psoriatic arthritis, and inflammatory bowel disease, or populations with chronically elevated biomarkers of inflammation may also benefit from similar therapeutic strategies to reduce inflammation." Clearly more research on the effects of disease-modifying therapy on cardiovascular risk in patients with systemic inflammatory disease is needed. Perhaps the exaggerated effects of inflammation in patients with these conditions will provide insights into disease mechanisms and therapeutic targets for atherosclerotic coronary disease in general.

The Education in Heart article (see page 397) this issue focuses on ischemic cardiomyopathy. This diagnosis implies significant left ventricular systolic dysfunction with an underlying pathophysiology that includes myocardial scarring, hibernation and stunning, or a combination of these disease states. The role of imaging in assessment of myocardial viability is emphasized (figure 3 ) with brief summaries of the role of echocardiography, single photon emission computed tomography (SPECT), positron emission tomography (PET), and magnetic resonance imaging (MRI). The effects of revascularization in patients with ischemic cardiomyopathy remain controversial. Instead, the key elements of evidence based therapy for ischemic cardiomyopathy are standard medical therapy for heart failure combined with implantable cardiac defibrillation (ICD) and/or biventricular pacing device therapy in appropriate patients.

The Image Challenge in this issue (see page 382) asks you to make the diagnosis from both a Doppler echocardiographic tracing and a chest computed tomographic (CT) image (figure 4) in a a 57 year old man after a motor vehicle accident. This case is not as simple as it might seem at first - see if you can make the correct diagnosis.

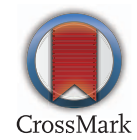

To cite Otto CM. Heart 2016;102:333-334.

Heart 2016;102:333-334.

doi:10.1136/heartjnl-2015-309220 